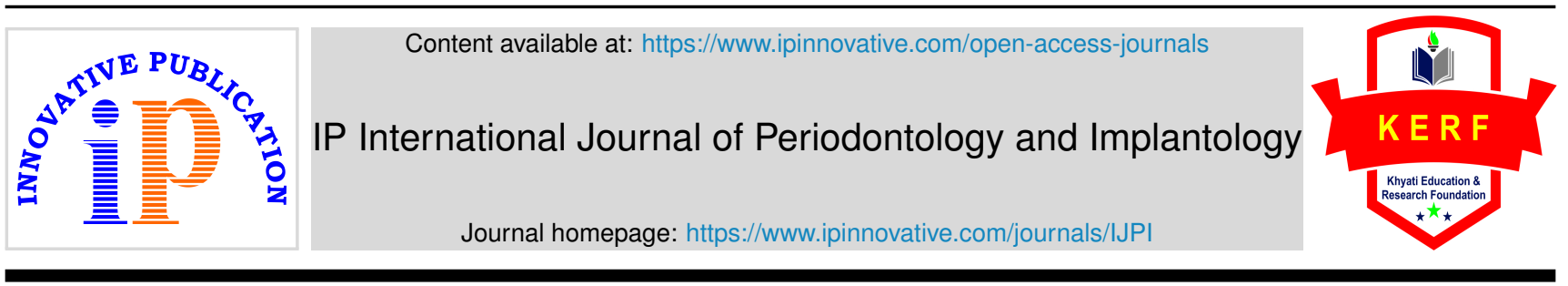

Original Research Article

\title{
Assessment of the awareness of periodontal diseases and oral hygiene practice among various healthcare professionals: A cross sectional study
}

\author{
Hrishikesh KP ${ }^{1}$,*, Jose Paul, Johnson Prakash D'lima, Senny Thomas Parackal, \\ Deepak Thomas, Ria Susan George
}

${ }^{1}$ Dept. of Periodontology, Annoor Dental College \& Hospital, Muvattupuzha, Kerala, India

\section{A R T I C L E I N F O}

\section{Article history:}

Received 20-11-2020

Accepted 26-11-2020

Available online 24-12-2020

\section{Keywords:}

Healthcare professionals

Oral hygiene

Awareness

Participants

\begin{abstract}
A B S T R A C T
Context: Oral health is an important component of general health that influences quality of life which affect appearance and psychological activities of person. Oral diseases have impact on overall systemic health. So doctor must play active role in educating their patients about role of oral diseases in their overall health

Aims: To assess the awareness of periodontal diseases and oral hygiene practice among healthcare professionals.

Settings and design: A total of 200 participants were selected from Ayurveda College, Homeopathic Medical College and two other hospitals for this study. Convenience sampling is used here.

Methods and Materials: 50 participants from each group were selected for study. The questionnaire consisted of 10 questions based on assessment of oral hygiene practice, knowledge of periodontal diseases and to promote oral hygiene.

Statistical analysis: Collected data was analyzed statistically using SPSS version 24 and utilized for statistical analysis. Pearson Chi-square test was used to evaluate differences between different variables and level of statistical significance was $\mathrm{P}<0.05$.

Results: Homeopathy participants were more concerned about their oral healthcare comparing other groups. About $62 \%$ of Ayurveda and Homeopathy participants were able to identify dental patients and refer them for proper dental care. All the groups have knowledge regarding periodontal diseases.

Conclusion: Although healthcare professionals who had undergone previous dental visit had some awareness regarding oral diseases, oral health knowledge was poor among healthcare professionals. There is also a need to enhance awareness of healthcare practitioners about periodontal disease.

(C) This is an open access article distributed under the terms of the Creative Commons Attribution License (https://creativecommons.org/licenses/by/4.0/) which permits unrestricted use, distribution, and reproduction in any medium, provided the original author and source are credited.
\end{abstract}

\section{Introduction}

Periodontal disease including gingivitis and periodontitis is an inflammation of the gums that results in loss of the supporting tissues holding the teeth in its socket. ${ }^{1}$ The signs and symptoms include reddish or swollen gums, bleeding gums, halitosis or in severe cases, loosening of teeth. Oral health diseases are harmful to the quality of life from childhood through old age and can have an effect on self-esteem, eating ability, diet and health. ${ }^{2,3}$ Oral

\footnotetext{
* Corresponding author.

E-mail address: hriz422@gmail.com (Hrishikesh KP).
}

health has been considered just equally as important as general health.More prevalence among the dental diseases are dental caries and the periodontal problem and it is a significant component of the burden of a global epidemic. ${ }^{4}$ The main cause of periodontal disease is bacterial plaque but many other factors such as hormonal changes, diabetes, poor diet, smoking and stress can affect initiation and progression. Oral health is an important component of general health that has been shown to influence the quality of life which can affect the appearance, social functions and day-to-day physical and psychological activities of the person. ${ }^{5}$ Oral diseases have a definite impact on overall 
systemic health; therefore, the doctor must play a more active role in educating their patients about the role of oral diseases in their overall health. ${ }^{1}$ But a healthcare professional's role in improving the population's oral health depends on his own knowledge of oral disease and its impact on general health, his attitude toward dentistry, and their daily practice in preserving oral health. The prevention of dental diseases requires complete involvement of the community, the professional and the individual. ${ }^{6}$ Previous literatures have reported that the healthcare professionals have deficit in knowledge regarding periodontal diseases.So the study was conducted to assess the awareness of periodontal disease and oral hygiene practice among various healthcare professionals. The reason for study being conducted in health care professionals is that they are directly involved with the society.

So if the healthcare professionals are aware about periodontal diseases and effective oral hygiene practices they can refer their patients to the dentist for diagnosis and treatment of periodontal diseases at early stage and also counsel their patients to maintain good oral hygiene.

\subsection{Subjects and Methods}

A total of 200 participants were selected from Government Ayurveda College and Hospital Thrippunithura, Dr Padiar Memorial Homeopathic Medical College Chottanikara, Indo American Brain and Spine Centre Chemmanakary and Medical Trust Hospital Ernakulam for this cross sectional study. The healthcare professionals include four groups: Ayurveda, Homeopathy, Physiotherapy and Nursing. 50 participants from each group were selected consisting of both males and females. The participants were briefed about the study and informed consent was obtained from them. The questionnaire consisted of 10 questions.

The questions were based on the assessment of oral hygiene practice, knowledge of periodontal diseases and to promote oral hygiene. Both male and female healthcare professionals who were interested to participate in the study were included. Institutional review board approval was obtained from Annoor Dental College and Hospital, Muvattupuzha, Kerala with Ref No: IHEC/08/20.

\section{Results}

Homeopathy participants were more concerned about their oral health care when compared to other groups of healthcare professionals. About $62 \%$ of Ayurveda and Homeopathy participants were able to identify dental patients and refer them for proper dental care when compared to other groups. All the groups have knowledge regarding periodontal diseases.

\section{Discussion}

The data was tabulated as graphs, based on the responses received.

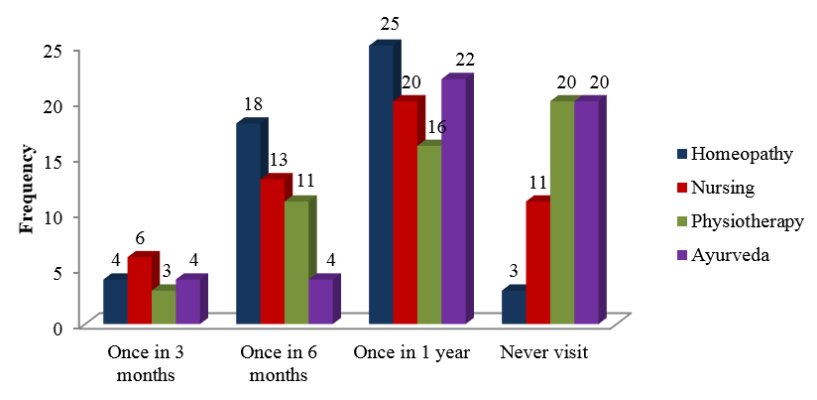

Fig. 1: How often do you visit the dentist for cleaning your teeth?

The above graphs revealed that most of the healthcare professionals visit dentists once a year and Homeopathy participants visit the dentists more frequently when compared to other groups.

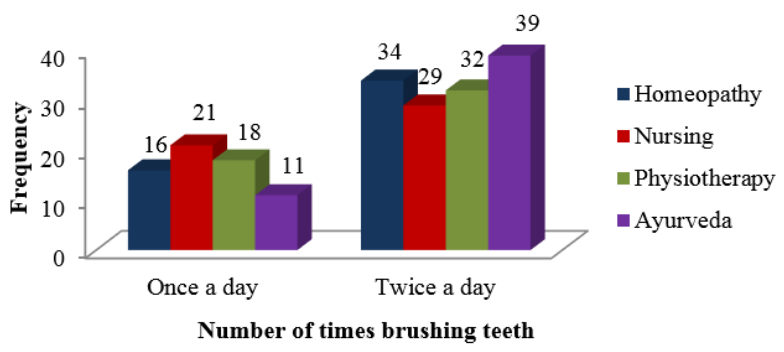

Fig. 2: How many times do you brush your teeth?

Among the homeopathy groups $68 \%$ participants brush their teeth twice a day while $58 \%$ of nursing, $64 \%$ of physiotherapy participants and $78 \%$ of Ayurveda participants brush their teeth twice a day.

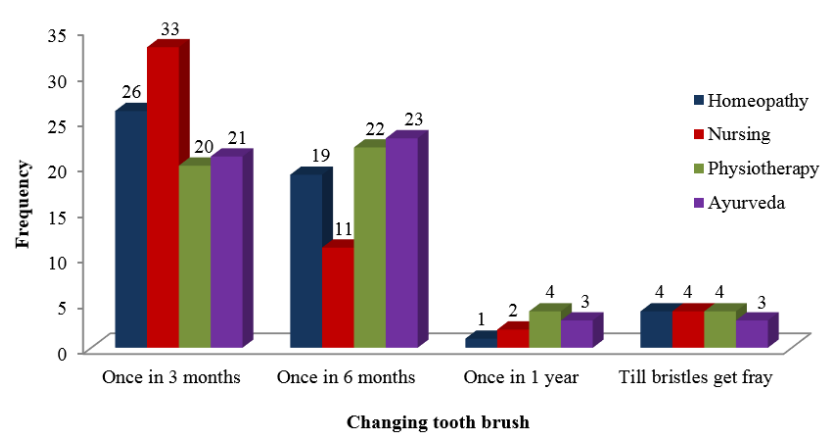

Fig. 3: How often do you change your tooth brush?

Most of the participants change their brush once in 3 months. 


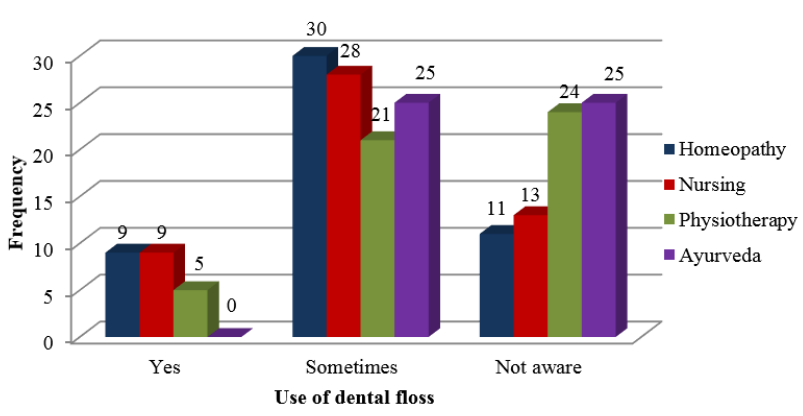

Fig. 4: Do you use dental floss?

More number of Homeopathy participants (78\%) use dental floss when compared to other groups. About 50\% participants in Ayurveda group are unaware of dental floss.

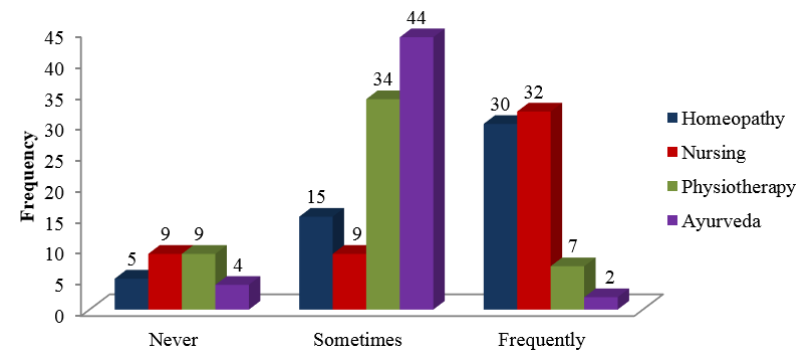

Fig. 5: Have you ever had patients with dental complaints?

About $88 \%$ of Ayurveda participants have encountered patients with dental complaint in their practice. While $82 \%$ of physiotherapy participants, $64 \%$ of nursing group and $60 \%$ of homeopathy participants have encountered patients with dental complaints in their practice.

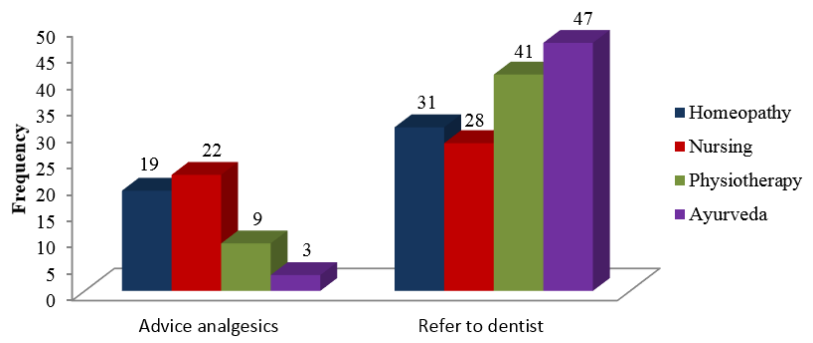

Fig. 6: If any of your patients is suffering from a sudden toothache, what will you do?

About $92 \%, 56 \%, 82 \%$ and $92 \%$ responses from Homeopathy, Nursing, Physiotherapy and Ayurveda respectively shows that they refer the patients to the dentist. Also some advice analgesics for the sudden toothache.

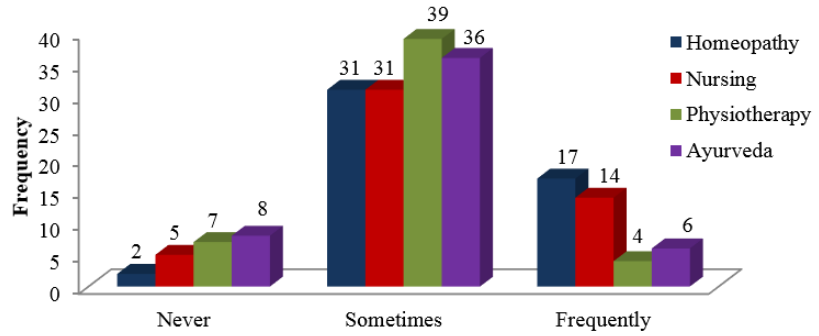

Fig. 7: Have you ever seen patients with oral lesions or conditions?

In Homeopathy group almost $96 \%$ participants came across patients with oral lesions or conditions. In Nursing, 90\% participants encountered patients with oral lesions or conditions. But only $68 \%$ participants in physiotherapy group came across patients with oral lesions or conditions. Nearly $84 \%$ of subjects from Ayurveda group have encountered patients with oral lesions or conditions.

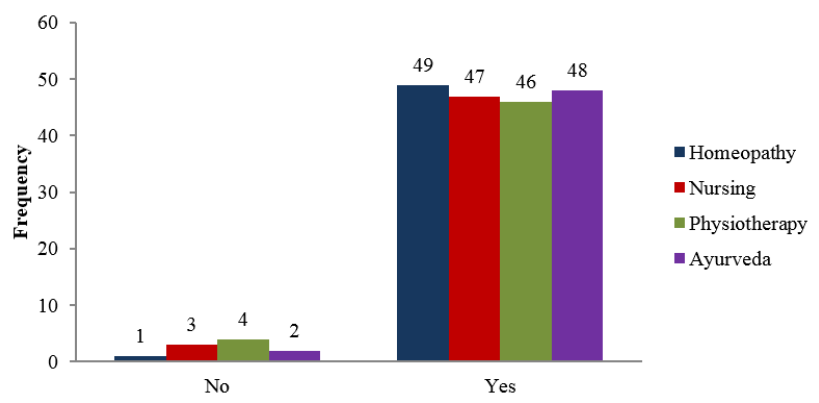

Fig. 8: Do you counsel patients for good oral hygiene?

Most of the healthcare professionals counsel their patients for good oral hygiene.

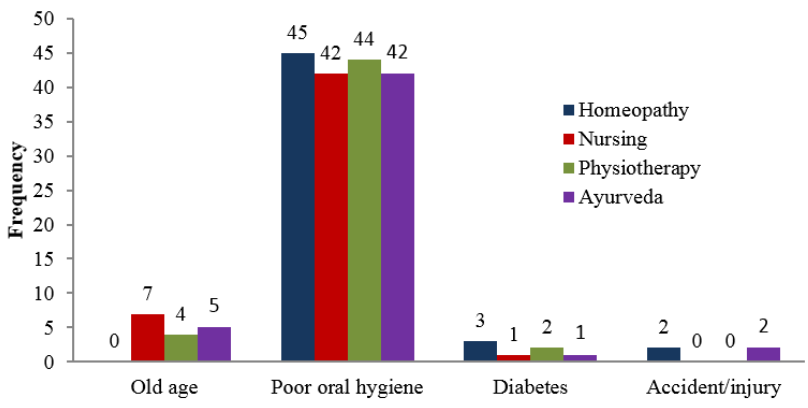

Fig. 9: What do you think is the commonest cause for loose teeth?

In the above graph $90 \%$ from Homeopathy, $84 \%$ from Nursing, $88 \%$ from Physiotherapy and $84 \%$ Ayurveda participants are supporting that the common cause for loose teeth is poor oral hygiene while a minor number of healthcare professionals reported that old age, diabetes and accident/injury can be common cause for loose teeth. 


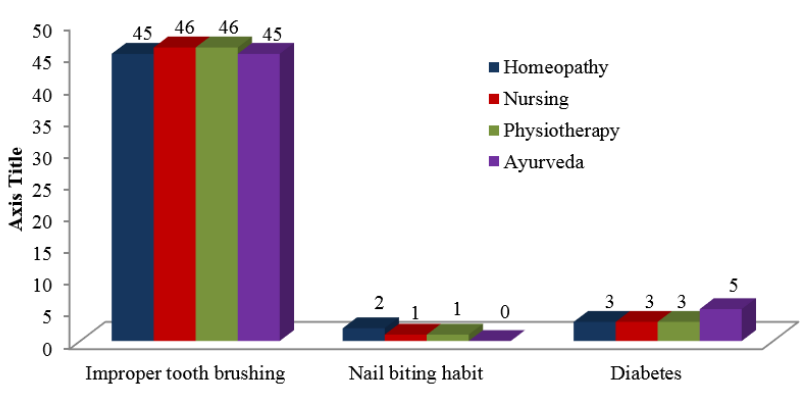

Fig. 10: What is the commonest cause for receding gums?

In the above graph $90 \%$ from Homeopathy, $92 \%$ from Nursing, 92\% from Physiotherapy and 90\% Ayurveda participants are supporting that the common cause for receding gums is improper tooth brushing. Almost $6 \%$ subjects from homeopathy, nursing and physiotherapy shows that diabetes causes the receding gums.

This study was conducted to assess the dental knowledge and awareness of different healthcare professionals. From the results it is apparent that periodontal disease awareness among healthcare professionals is good. Several studies have evaluated the oral health knowledge, attitude, and oral hygiene practice among health care providers. According to the study by Deinzer et al., showed that knowledge deficit was seen in all topics investigated in the given population. ${ }^{7}$ Bhavya B et al., reported that majority $(90.9 \%)$ of respondents had positive attitudes toward the referral of their patients for regular periodontal care. ${ }^{8}$

They must be knowledgeable to educate and counsel the patients, since oral health has an effect on the quality of the life and its association with the general systemic health. Educating the public regarding the importance of maintaining good oral hygiene and lack of maintaining good oral hygiene which causes oral and systemic diseases allow them to understand better and motivates them to preserve good oral hygiene. The impact of the oral disease on systemic health must be known to create awareness among the patients to maintain both oral and general systemic health.

A dental screening program may be required to be conducted for the healthcare professionals thus they can know their own oral health. It motivates healthcare professionals to maintain good oral hygiene. Attending awareness programs regarding oral health helps them to gain the knowledge and interest in the oral health.

These awareness programs can motivate them to learn about oral diseases and made them to educate the patients to maintain a good oral hygiene such as brushing technique and using other oral hygiene aids. ${ }^{9}$

Since the prevalence of periodontitis is one of the world's epidemics and because of its relation to systemic diseases, healthcare professionals can play an important role in diagnosing the disease and limit the proportion of the disease. ${ }^{10}$
Limitation of the study is that the above selected sample size is not truly representative of proportions in the healthcare community.

\section{Conclusions}

Although healthcare professionals who had undergone previous dental visit had some awareness regarding the oral diseases, oral health knowledge was poor among the healthcare professionals. There is also a need to enhance the awareness of health care practitioners about the periodontal disease. Maintenance of good oral hygiene should be incorporated in their curriculum. One of the main disadvantage of the study is the small sample size. Further cross sectional studies on a larger sample size will help us in understanding the need to improve their knowledge and awareness of healthcare professionals.

\section{Source of Funding}

No financial support was received for the work within this manuscript.

\section{Conflict of Interest}

The authors declare they have no conflict of interest.

\section{References}

1. Lang NP, Bartold PM. Periodontal health. J Periodontol. 2018;89:S916. doi:10.1002/1per.16-0517,

2. Walker A, Cooper I. Adult dental health survey: oral health in the United Kingdom 1998. Stationery Office; ; 1998.

3. Chen MS, Andersen RM, Barmes DE, Leclerq MH, Lyttle CS. Comparing oral health care systems: a second international collaborative study. World Health Organization; 1997.

4. Dagli RJ, Tadakamadla S, Dhanni C, Duraiswamy P, Kulkarni S. Self reported dental health attitude and behavior of dental students in India. J Oral Sci. 2008;50(3):267-72. doi:10.2334/josnusd.50.267.

5. Gomes AS, Abegg C, Fachel JG. Relationship between oral clinical conditions and daily performances. Braz Oral Res. 2009;23:76-81. 101:10.1590/s $1806-83242009000100013$.

6. Locker D. Measuring oral health: a conceptual framework. Community Dent Health. 1988;5:3-18

7. Deinzer R, Micheelis W, Granrath N, Hoffmann T. More to learn about: periodontitis-related knowledge and its relationship with periodontal health behaviour. J Clin Periodontol. 2009;36(9):756-64. d01:10.1111/.1600-051x.2009.01452.x

8. Bhavya B. Knowledge, practice and attitude of medical doctors on the association between medical health and periodontal health. $J$ Dent Oro-facial Res. 2015;11(1).

9. Smadi L, Nassar OS. Oral \& Dental Health Knowledge and Attitude among Nursing Students. World J Educ Res. 2016;3(2):238-50. doi:10.22158/wjer.v3n2p238.

10. Susmitha E. Assessment of knowledge about periodontal disease among a group of health care professionals in Chennai city. Asian J Pharmaceuticals Clin Res. 2015;8:101-5.

\section{Author biography}

Hrishikesh KP, Post Graduate Student 
Jose Paul, Professor and HOD

Johnson Prakash D'lima, Professor

Senny Thomas Parackal, Professor

Deepak Thomas, Reader

Ria Susan George, Senior Lecturer
Cite this article: Hrishikesh KP, Paul J, D'lima JP, Parackal ST,

Thomas D, George RS. Assessment of the awareness of periodontal diseases and oral hygiene practice among various healthcare professionals: A cross sectional study. IP Int J Periodontol Implantol 2020;5(4):149-153. 